\title{
3-D numerical simulations of coronal loops oscillations
}

\author{
M. Selwa $a^{1,2}$ and L. Ofman ${ }^{1,2,3}$ \\ ${ }^{1}$ Department of Physics, The Catholic University of America, 620 Michigan Avenue, NE, 200 Hannan Hall, Washington, \\ D.C., 20064, USA \\ ${ }^{2}$ NASA Goddard Space Flight Center, Code 671, Greenbelt, MD 20771, USA \\ ${ }^{3}$ Visiting Associate Prof. Dept. of Geophysics \& Planetary Sciences Tel Aviv University, Ramat Aviv, Tel Aviv, Israel
}

Received: 1 June 2009 - Revised: 27 July 2009 - Accepted: 20 August 2009 - Published: 13 October 2009

\begin{abstract}
We present numerical results of 3-D MHD model of a dipole active region field containing a loop with a higher density than its surroundings. We study different ways of excitation of vertical kink oscillations by velocity perturbation: as an initial condition, and as an impulsive excitation with a pulse of a given position, duration, and amplitude. These properties are varied in the parametric studies. We find that the amplitude of vertical kink oscillations is significantly amplified in comparison to horizontal kink oscillations for exciters located centrally (symmetrically) below the loop, but not if the exciter is located a significant distance to the side of the loop. This explains why the pure vertical kink mode is so rarely observed in comparison to the horizontally polarized one. We discuss the role of curved magnetic field lines and the pulse overlapping at one of the loop's footpoints in 3-D active regions (AR's) on the excitation and the damping of slow standing waves. We find that footpoint excitation becomes more efficient in 3-D curved loops than in 2-D curved arcades and that slow waves can be excited within an interval of time that is comparable to the observed one wave-period due to the combined effect of the pulse inside and outside the loop. Additionally, we study the effect of AR topology on the excitation and trapping of loop oscillations. We find that a perturbation acting directly on a single loop excites oscillations, but results in an increased leakage compared to excitation of oscillations in an AR field by an external source.
\end{abstract}

Keywords. Solar physics, astrophysics, and astronomy (Corona and transition region) - Space plasma physics (Kinetic and MHD theory; Waves and instabilities)

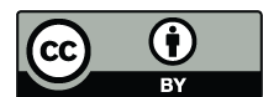

Correspondence to: M. Selwa (malgorzata.a.selwa@nasa.gov)

\section{Introduction}

Observations with recent instruments such as SUMER onboard SOHO and TRACE in EUV wavelength resulted in the detection of various oscillations in solar coronal loops. Among them several kinds of waves have been identified, e.g. propagating (De Moortel et al., 2002) and standing (Wang et al., 2002) slow magnetosonic waves. Wang et al. (2003a, b) reported detailed statistical study of several events where waves were detected. The observations show that slow standing waves excitation is related to footpoint brightening in coronal loops. The properties of slow standing waves were described by Wang et al. (2005). Other observations were interpreted as evidence for fast sausage waves (Nakariakov et al., 2003; Aschwanden et al., 2004) as well as different polarizations of fast kink magnetosonic waves: horizontal (Nakariakov et al., 1999; Aschwanden et al., 1999, 2002; Schrijver et al., 2002) and vertical (Wang and Solanki, 2004). Recently, high cadence STEREO EUVI observations of the quiet sun (QS) (Patsourakos and Vourlidas, 2007) and AR's (Verwichte et al., 2009) found additional evidence of coronal loop oscillations. Recently Wang et al. (2008) reconsidered oscillations described by Aschwanden et al. (2002) and found that out of three oscillations previously identified as fundamental horizontal mode oscillations, two cases appeared to be fundamental vertical mode oscillations (but possibly combined with the fundamental horizontal mode), and one case appeared to be a combination of the fundamental vertical and horizontal modes, while in three cases it was not possible to clearly distinguish between the fundamental mode and the second harmonic of the horizontal oscillation. In five other cases it was not possible to clearly distinguish between a fundamental horizontal mode and the second-harmonic of a vertical mode. Wang et al. (2008) study concluded that pure modes are observed less often than the combination of different polarizations, and that pure vertical coronal loop oscillations happen less often than pure horizontal ones.

Published by Copernicus Publications on behalf of the European Geosciences Union. 
One common feature of these observations is that the decay of the oscillations is very rapid. Several mechanisms were suggested as an explanation of the fast damping of transverse loop oscillations, e.g. enhanced viscosity (Nakariakov et al., 1999), wave leakage (Smith et al., 1997; Selwa et al., 2007a), phase mixing (Ofman and Aschwanden, 2002) and resonant absorption (Ruderman and Roberts, 2002). More detailed theoretical description of the damping mechanisms and other kink waves properties can be found in Ruderman and Erdélyi (2009).

Two polarizations of kink oscillations were studied theoretically by Van Doorsselaere et al. (2004). The authors solved linearized ideal MHD equations in zero $\beta$ regime and found that quasi-mode frequencies are unchanged up to first order in the curvature. The imaginary part of the frequency, however, does change in first order, and quasi-modes are slightly more damped in realistically curved coronal loop configurations compared to loops modeled as straight cylinders. Since vertical oscillations are confined to a single plane, there are several 2-D numerical studies of vertical oscillation properties in curved loops, e.g. Selwa et al. (2005b, 2006, 2007a). The full 3-D resistive MHD simulation of simultaneously excited vertical and horizontal oscillations were performed by McLaughlin and Ofman (2008). That model included several approximations, relaxed in the present study. The main approximation is that the high density loop in the AR has constant cross section, and does not expand with height, as expected for a flux tube in a dipole field. Thus, the oscillation modes of such a loop are not eigenmodes of a particular flux-tube.

There are several unresolved questions about the nature of loops oscillations. One problem is why only a small fraction of TRACE flare observations show oscillating loops. Another open question is the excitation mechanism of vertical and horizontal kink oscillations, and in particular it is not clear why most of detected oscillations are horizontally polarized. The last question considered here, concerns slow standing waves and their excitation mechanism within single wave-period. The present study addresses these questions by performing 3-D simulations of loop oscillations, and showing that the topology of the field and the location of the flare in the AR have strong effect on loop oscillations.

There are several studies of waves in coronal loops that use 3-D MHD model, including several damping mechanisms as well as other important features summarized below. For the detailed review see Ofman (2009a). Propagating waves were studied by Selwa et al. (2004). Terradas and Ofman (2004) studied density enhancements produced nonlinearly by loop oscillations using 1-D, 2-D and 3-D MHD models. Ofman $(2005,2009 \mathrm{~b})$ studied kink and fast magentosonic waves and presented several 3-D models of the loops including straight cylinder and 4 cylindrical straight and twisted loops. McLaughlin and Ofman (2008) studied excitation of waves in 3-D cylindrical curved loop. Ofman and Thompson (2002a) and Ofman (2007) studied wave be- havior in 3-D active regions with gravity based on a dipole field and magnetogram data, respectively. However, their models did not include a denser loop structure, which is necessary to examine behavior of trapped modes in a single loop.

Numerical studies of slow standing waves concentrate mainly on explaining the process of excitation and attenuation of the slow standing waves. For instance, Ofman and Wang (2002) showed that nonlinear slow standing waves are strongly damped by thermal conduction. Mendoza-Briceño et al. (2004) discussed the effect of gravity on attenuation of slow standing waves. Selwa et al. (2005a) showed that footpoint leakage may be an efficient damping mechanism of slow standing waves. This result was also confirmed by Ogrodowczyk and Murawski (2007). Selwa et al. (2007a) reported that lateral leakage can also lead to damping of slow standing waves. Recently Sigalotti et al. (2007) studied dissipation of slow standing waves by thermal conduction, compressive viscosity, radiative cooling, and heating for nonstratified and stratified loops.

Excitation of slow standing waves was numerically modeled by Nakariakov et al. (2004) and Tsiklauri et al. (2004) who showed that only the second harmonic is excited, regardless of a spatial position of heat deposition. However, Selwa et al. (2005a) showed that pulses close to a footpoint excite the fundamental mode of the slow standing wave, while pulses close to the apex excite the second harmonic. Additionally, these authors studied the influence of the pulse and loop parameters on the excitation and attenuation of slow standing waves. Taroyan et al. (2005) also showed that the observed slow standing waves may have their origin in footpoints of the loop, however the duration of the pulse was too long to be in agreement with observations (one wave period) and depended strongly on the temporal profile of the pulse. Selwa et al. (2006) results showed that footpoint excitation in a curved loop may lead to excitation of the fundamental slow standing mode, while excitation below the apex of the loop leads to the excitation of the first harmonic. Ogrodowczyk and Murawski (2007) found that slow waves are excited faster and attenuated more efficiently in 2D straight slab than in 1-D loop due to coupling between the fast and slow magnetosonic waves. Selwa et al. (2007b) reported the excitation of slow standing mode in a 2-D curved arcade loop within 1.6 wave-periods from the heat pulse. Recently Haynes et al. (2008) showed that the kink instability in a straight loop initially sets up the second mode that is converted through the rearrangement of the magnetic field into two out-of-phase fundamental slow modes. These slow modes are in the two entwined flux tubes created during the kink instability. However, this excitation mechanism leads to fundamental standing mode after $\sim 2$ wave-periods, so this model still does not fully explain the observations.

In this paper we extend the Ofman and Thompson (2002) and McLaughlin and Ofman (2008) models by modeling a dipolar AR with a flux tube denser than the surrounding corona. Our model includes the effect of non-constant 
cross-section of the loop due to flux tube expansion with height, and provides an approximation of realistic AR loop oscillation, where such expansion is present.

The paper is organized as follows: the numerical model is described in Sect. 2. The numerical results are presented in Sect. 2.1. This paper is concluded by a short summary of the main results in Sect. 3.

\section{Numerical model}

We model the solar coronal plasma with the normalized, three-dimensional, nonlinear, resistive MHD equations:

$$
\begin{aligned}
\frac{\partial \varrho}{\partial t}+\nabla \cdot(\varrho \mathbf{V}) & =0, \\
\varrho\left[\frac{\partial \mathbf{V}}{\partial t}+(\mathbf{V} \cdot \nabla) \mathbf{V}\right] & =-E_{u} \nabla p+(\nabla \times \mathbf{B}) \times \mathbf{B}, \\
\frac{\partial \mathbf{B}}{\partial t} & =\nabla \times(\mathbf{V} \times \mathbf{B})+\frac{1}{S} \nabla^{2} \mathbf{B} .
\end{aligned}
$$

Here $\varrho$ denotes the mass density, $\mathbf{V}$ is plasma velocity, $\mathbf{B}$ is divergence free $(\nabla \cdot \mathbf{B}=0)$ magnetic field vector, $p$ is plasma pressure. Euler number, $E_{u}$ and Lundquist number, $S$, are given as:

$$
\begin{aligned}
E_{u} & =\frac{\beta}{2}=\frac{c_{\mathrm{s}}^{2}}{\gamma V_{\mathrm{A} 0}^{2}}, \\
S & =\frac{L_{s} V_{\mathrm{A} 0}}{\eta},
\end{aligned}
$$

where plasma $\beta$ is the ratio of the thermal to the magnetic pressures, $c_{\mathrm{S}}$ and $V_{\mathrm{A} 0}$ denotes sound and Alfvén speed, respectively. $L_{s}$ is a typical length scale in the system and $\eta$ denotes resistivity. We neglect viscosity and gravity in the model presented here. For simplicity we study the isothermal case $(\gamma=1)$ and therefore $p=n k_{B} T_{0}$, eliminating the need to solve the energy equation. Here $n$ is particle density, $k_{B}$ denotes Boltazmann's constant and $T_{0}$ is plasma temperature. This assumption is justified since most of TRACE loops were observed to be nearly isothermal.

We take the magnetic field strength of the dipole as $B_{0}=93$ Gauss, temperature $T_{0}=1 \mathrm{MK}$ and density $n_{0}=\varrho_{0} / m_{p}=10^{9} \mathrm{~cm}^{-3}$, where $m_{p}$ is the proton mass. These values correspond to the Alfvén speed $V_{\mathrm{A} 0}=6414 \mathrm{~km} / \mathrm{s}$, the isothermal sound speed $c_{s}=128.5 \mathrm{~km} / \mathrm{s}$ and Alfvén time $\tau_{\mathrm{A}}=10.9 \mathrm{~s}$. Furthermore, the spatial unit in our normalization is $L_{s}=R_{s} / 10=69.55 \mathrm{Mm}$, where $R_{s}$ is solar radius. For the cylindrical loop we hold the density and temperature fixed while we choose magnetic field strength as $B_{0}=15$ Gauss to make the plasma $\beta \leq 0.1$ and magnetic field strength at the apex of the dipole loop comparable to cylindrical loop midpoint values.

\section{Numerical code}

We use the numerical code NLRAT that implements the modified Lax-Wendroff scheme with a fourth-order smoothing term for solving time-dependent resistive isothermal MHD equations. The solenoidality condition $(\nabla \cdot \mathbf{B}=0)$ is satisfied by using Powell's (1994) method. More detailed description of the code can be found in Ofman and Thompson (2002).

Equations (1-3) are solved numerically in an Eulerian box with the $x-, y$ - and $z$-dimensions $\left(x_{\min }, x_{\max }\right) \times\left(y_{\min }, y_{\max }\right) \times\left(z_{\min }, z_{\max }\right)=(-3.5,3.5) \times$ $(-3.5,3.5) \times(3,6.5)$. All spatial coordinates are given in units of $L_{s}$. This box is covered by a uniform grid of $150 \times 150 \times 150$ numerical cells. Grid convergence studies showed that this resolution is sufficient to get results independent of the grid.

We apply open boundary conditions, with a zero-gradient extrapolation of all plasma variables at all the boundaries except $z_{\min }$ photospheric boundary to allow a wave signal to leave freely the simulation region. At the bottom of the simulation region $\left(z_{\min }\right)$ we model wave reflection from photospheric layer for $z<z_{\min }$ by keeping magnetic field, velocity and density fixed:

$\mathbf{B}\left(x, y, z_{\min }, t\right)=\mathbf{B}_{0}\left(x, y, z_{\min }\right)$,
$\mathbf{V}\left(x, y, z_{\min }, t\right)=0$,
$\varrho\left(x, y, z_{\min }, t\right)=\varrho_{0}\left(x, y, z_{\min }\right)$.

\section{Initial conditions}

As the initial equilibrium magnetic field of our active region we take an idealized 3-D potential dipole. Magnetic fieldlines are shown in the right panel of Fig. 1. Detailed analytical expressions describing this magnetic configuration can be found in Ofman and Thompson (2002). For simplicity we assume a constant equilibrium density. The loop density is 3 times larger than in the surrounding AR plasma (see below).

\section{The loop}

TRACE observations reveal the presence of dense loops in the corona on small and large scale (compared to AR case). Following McLaughlin and Ofman (2008) we include a denser loop in our model by filling a flux tube with denser plasma than in the surrounding AR (Fig. 1). Note that such a loop has lower Alfvén speed in comparison to the ambient corona and works as a waveguide in the system, capable of trapping waves. It is important to note that individual coronal loops appear to have a constant cross section (e.g., Klimchuk, 2000; López Fuentes et al., 2006, 2008). However, a collection of loops do expand with height in a manner consistent with expanding flux tube in our model (López Fuentes et al., 2008). Thus, our loop can be viewed as an unresolved collection of small scale loops.

While McLaughlin and Ofman (2008) increased density inside the loop was defined by a sharp gradient at the loop 

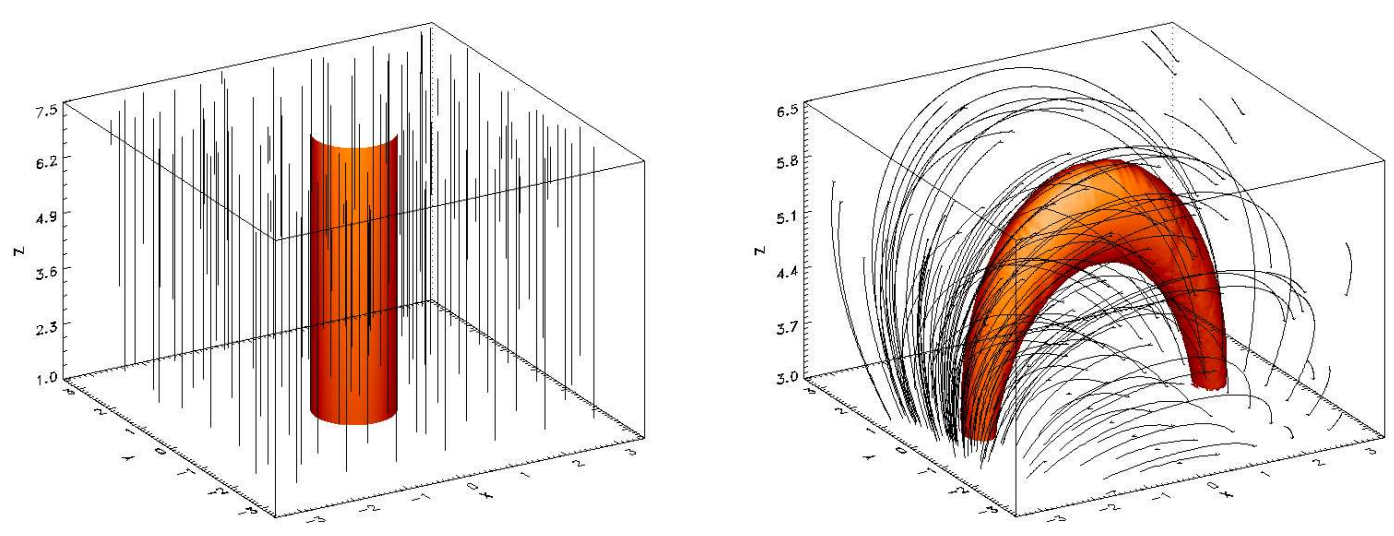

Fig. 1. Initial 3-D magnetic field configuration of the model AR with the high density loop as a flux-tube for straight (left panel) and dipole (right panel) fields. Spatial coordinates are measured in units of $L_{S}$.

boundary, we use smooth density profile which is closer to observations (Aschwanden et al., 2003):

$\varrho_{\mathrm{i}}=\varrho_{\mathrm{e}} d \exp \left[-\frac{\left(y-y_{0}\right)^{2}+\left(z-z_{0}\right)^{2}}{w}\right]$.

Here subscripts i(e) denote the loop (ambient corona), $\left(x_{0}, y_{0}, z_{0}\right)=(0,0,5.5)$ denotes the central point of the fieldline chosen as the axis of mass loaded loop, $w=2 r=0.375$ is the loop width at the apex and $d=3$ denotes density ratio between the loop and ambient corona. The contrast value is chosen to be in agreement with observations of loops oscillating in horizontal kink mode (Aschwanden et al., 2003) and in vertical kink mode (Selwa et al., 2007). The length of the loop was chosen to be $\sim 7 L_{s}$.

We initialize the high density loop in the flux-tube by tracing field lines starting in the $y-z$ plane at $x=0$. Each of the fieldlines is parameterized with a factor associated with the value of Gaussian density profile (Eq. 9) along the whole flux tube. From the momentum Eq. (2) in equilibrium it follows that for such an equilibrium configuration the sum of Lorentz force and thermal pressure gradient force must vanish:

$E_{u} \nabla p-(\nabla \times \mathbf{B}) \times \mathbf{B}=0$.

Since the initial magnetic field is potential $(\nabla \times \mathbf{B})=0$ and plasma $\beta$ (Euler number) is small in the system, the density variation across the loop does not affect significantly the force balance. For this reason we can choose the density distribution across the fieldlines (e.g., flux-tube width that varies with height) to satisfy Eq. (9). In equilibrium the increased pressure inside the loop is balanced by the magnetic pressure gradient. In low- $\beta$ coronal loop, the perturbation due to the increased loop density is small. Consequently, our loop is near equilibium state at $t=0$. However, we expect small adjustments of the loop structure in the initial stage of the simulation, as the small non-potential component of the magnetic field is produced to balance the small thermal pressure at the flux-tube boundary.
The two kinds of model ARs we consider are displayed in Fig. 1. The color features show the isosurface of density within the denser loop while black lines represent the magnetic fieldlines. Note the difference in fieldline length and orientation for the two kinds of ARs.

\section{The perturbation}

We perturb our equilibrium model with two forms of excitation. One form is the nearly eigenmode velocity profile corresponding to the Gaussian loop profile launched inside the loop. Alternative perturbation form is external velocity pulse at $x-z$-boundary plane along $y_{\min }$, which models the impact of fast magnetosonic wave on our active region:

$$
\begin{aligned}
V_{y}= & A_{V} V_{\mathrm{A} 0} \exp \left[-\left(\frac{x-x_{0}}{w}\right)^{2}\right] \\
& \times \exp \left[-\left(\frac{z-z_{0}}{w}\right)^{2}\right] \\
& \times \exp \left[-\left(\frac{2 t-\left(t_{1}+\frac{t_{2}-t_{1}}{2}\right)}{t_{2}-t_{1}}\right)^{8}\right] .
\end{aligned}
$$

Here $A_{V}=0.1$ (cylindrical loop) and $A_{V}=0.025$ (dipole loop) is the relative amplitude of the pulse, $V_{\mathrm{A} 0}$ is the maximum Alfvén speed at the bottom of the simulation region, $t_{1}=2.5 \tau_{A}, t_{2}=5 \tau_{A} x_{0}=0, z_{0}=z_{\max } / 2$ and $w=0.7$.

Additionally, we perturb the loop from below by launching a Gaussian velocity pulse at $x-y$ boundary plane (e.g. left top panel of Fig. 2), depending on the problem.

\subsection{Numerical results}

\subsubsection{Vertical vs. horizontal oscillations}

We start our studies with the loop shown in right panel of Fig. 1. At the beginning we perturb our loop with the 

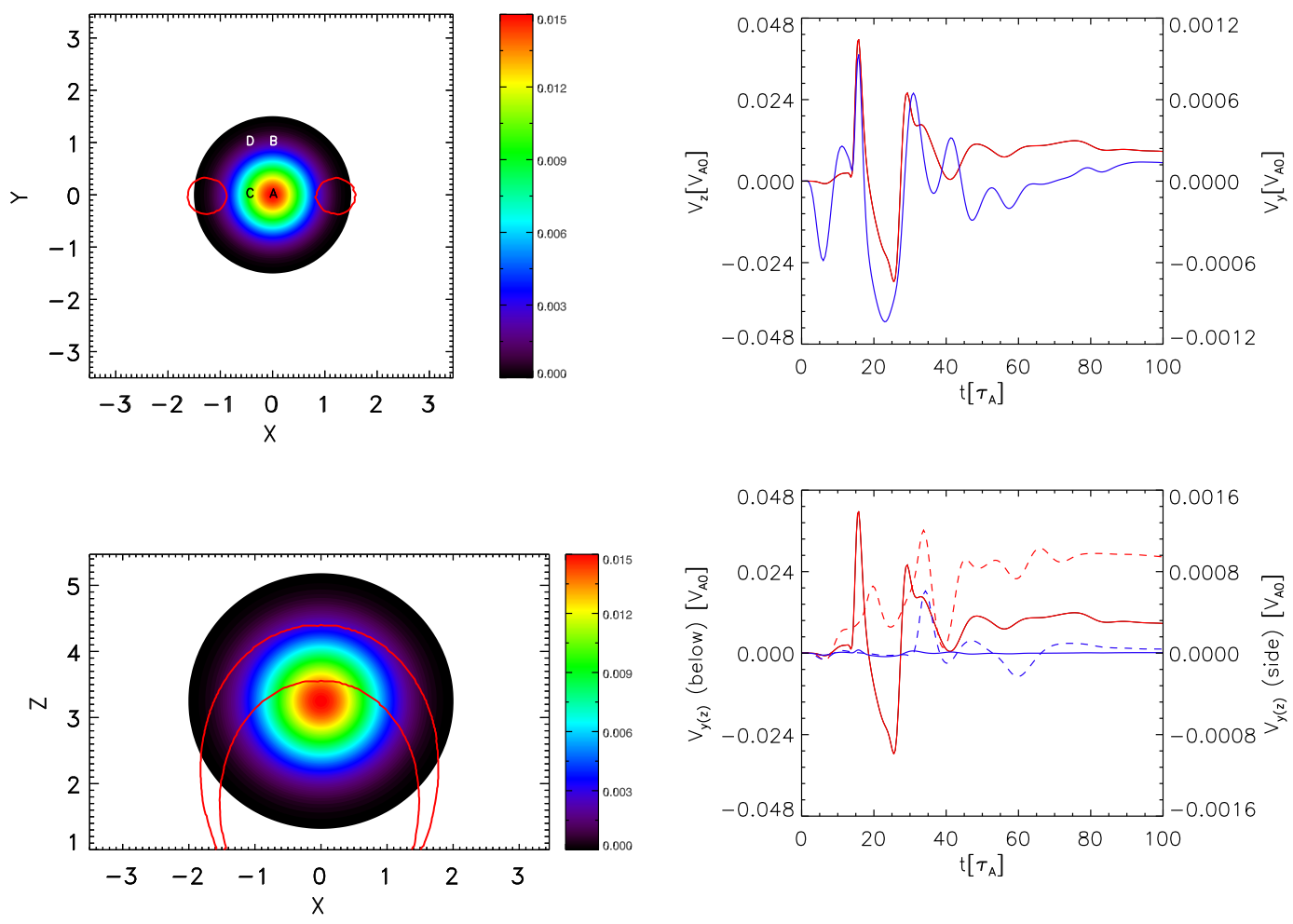

Fig. 2. Left panels: Spatial profiles of the pulses launched in $z$ component of velocity (top panel, $x y$ plane) and in $y$ component of velocity (bottom panel, $x z$ plane) (color scales). Red contours display the initial position of the loop (different plane than the pulse in bottom panel). Letters in a top panel show various positions of the center of the pulse. Spatial coordinates are given in units of $L_{s}$. Right panels: Time signatures of $z(y)$ component of velocity at the loop apex (red (blue) lines, respectively). Top panel corresponds to the excitation by the pulse shown in top panel of this figure (note difference in scales for $V_{z}$ and $V_{y}$ ). Bottom panel corresponds to the excitation by the pulse shown in top panel (solid lines) and by the pulse shown in bottom panel of this figure (dashed lines), respectively. Note difference in $v_{Z}$ to $v_{Y}$ amplitude ratio for both kinds of excitation.

Gaussian pulse acting below the loop (presented in left top panel of Fig. 2). The position of the center of the exciter varies (different locations are marked with letters) in parametric studies. The position of the pulse does not change difference images of the oscillations (not shown) and leads to vertical oscillations of the loop. However, the ratio of maximum amplitude of vertical to horizontal component of velocity, $V_{z} / V_{y}$, at the loop's apex varies with the position of the pulse. For the pulses centered along $y_{0}=0$ line (below the loop's apex) the ratio of amplitudes is close to $\sim 35$, while it decreases to $\sim 7$ with the pulse center moved to $y_{0}=1$ line. It is noteworthy that moving the position along $y_{0}$ line does not affect the results (the ratio of maximum velocity amplitudes for the pulse (A) is the same as for the pulse (C) and for pulses (B) and (D) is also the same). Typical time signature of $V_{z}$ (red line) and $V_{y}$ (blue line) in case of pulse (A) is shown in the top panel of Fig. 2. The pulse reaches the detection point (the midpoint of the apex of the loop) at $t=15$ and excites both horizontal $\left(V_{y}\right)$ and vertical $\left(V_{z}\right)$ oscillations. The small amplitude motions before $t=15$ are due to the adjustment phase (Ofman, 2007; McLaughlin and Of- man, 2008) as we do not start the simulations from a strict equilibrium. Due to the very small amplitude of horizontal oscillation in comparison to the vertical one this oscillation is very unlikely to be observed compared to clearly seen vertical oscillation (note the difference in scales in left bottom panel of Fig. 2).

Switching to the excitation by the pulse from the side (right top panel of Fig. 2) changes the scenario. Most of the observed kink oscillations are horizontal oscillations (Nakariakov et al., 1999; Aschwanden et al., 1999; Schrijver et al., 2002). The next question that arises is: why they are so easily excited in comparison to vertical ones? We showed that moving the pulse to the side of the loop (but still acting below the loop) can excite both vertical and horizontal oscillation with the amplitude ratio lower than one order of magnitude. The pulse acting below, but far away from the loop may be modeled in the limit of distant pulse as the pulse from the side boundary (as suggested McLaughlin and Ofman, 2008). However, we modify it by using the Gaussian shape pulse centered below the loop's apex (right top panel of Fig. 2). It is noteworthy that in such a case the maximum 

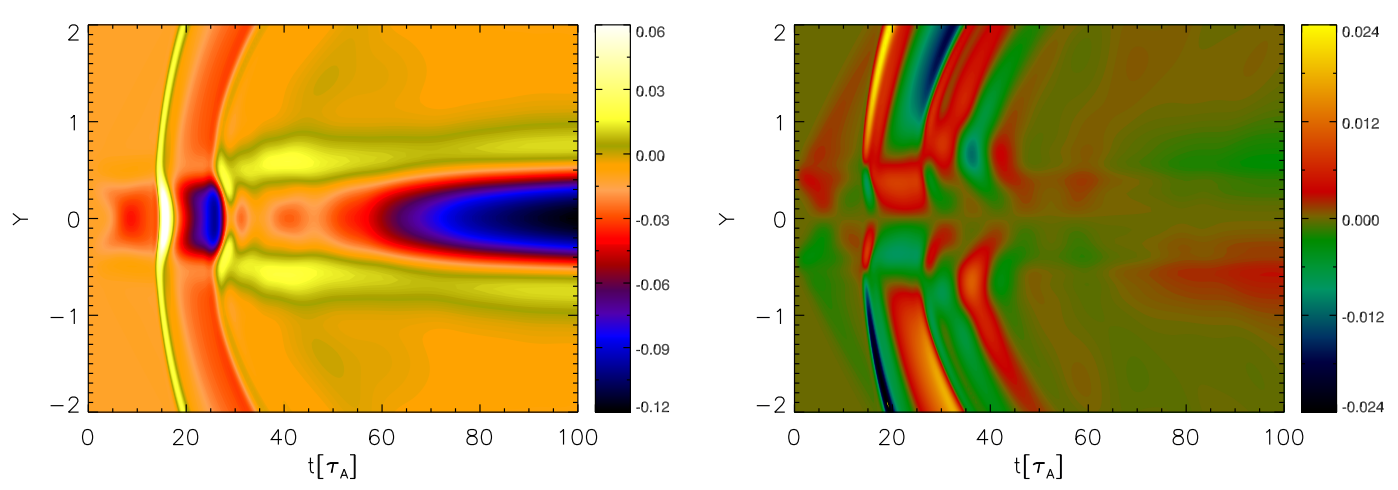

Fig. 3. Time signatures along vertical axis of the loop $(x=y=0)$ of perturbed mass density (left panel, color scale, units of $\left.\varrho_{0}\right)$ and $y$ component of velocity (right panel, color scale, units of $V_{\mathrm{A} 0}$ ) in case of excitation with the pulse (A) shown in top panel of Fig. 2 . The loop is centered around $y=0$. Spatial coordinates are given in units of $L_{S}$.
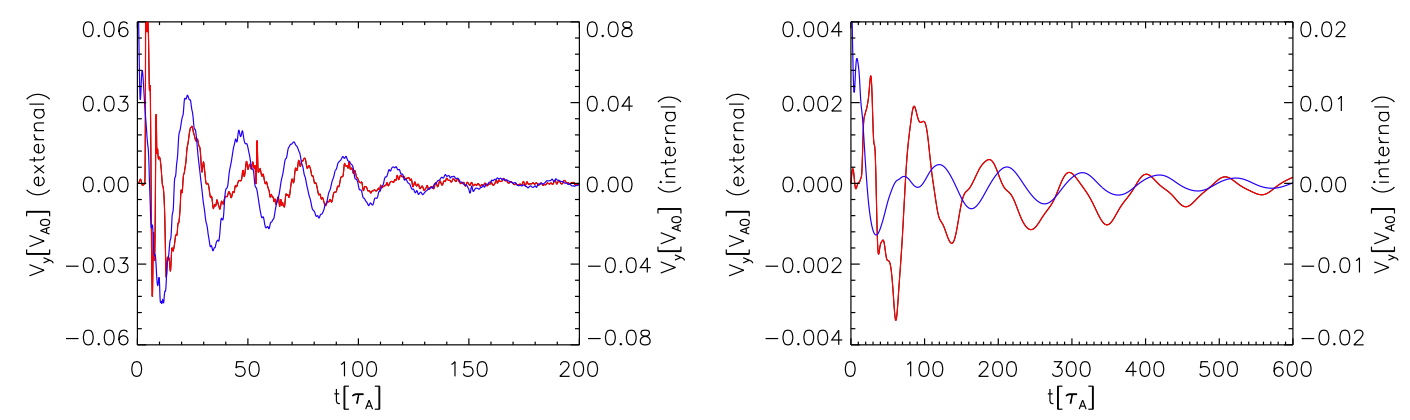

Fig. 4. Time signatures of transversal component of velocity at the cylindrical (left panel) and dipole (right panel) loop's apex. Blue lines correspond to the excitation by the pulse inside the loop while red lines show the evolution after external excitation.

amplitudes in vertical component of velocity, $V_{z}$, (red lines) and horizontal one, $V_{y}$, (blue lines) are of the same order of magnitude (dashed lines) in contrast to the case of symmetric pulse below the loop (case A, left top panel of Fig. 2). Such a pulse is likely to excite a combination of global horizontal and vertical oscillation.

As a possible damping mechanism we suggest energy leakage (Smith et al., 1997). It was found by Selwa et al. (2005b, 2007a) that vertical oscillations in 2-D arcade loop are damped through energy leakage and confirmed by McLaughlin and Ofman (2008) in 3-D geometry. Selwa et al. (2005b, 2007a) detected short period (compared to global kink mode period) waves propagating out of the loop in the direction of decreasing Alfvén speed (above the loop) in the form of sausage mode waves. In 3-D geometry the scenario may be more complicated and energy leakage in the form of horizontal sausage mode takes place (Fig. 3) and serves as the damping mechanism for the loop oscillations.

\subsubsection{AR topology and kink oscillations}

We start our studies with the perturbation of AR loops from outside with a time dependent side boundary pulse. The corresponding time signatures are displayed in Fig. 4 with red lines. In both cases we observe similar behavior of the loops: initial perturbation is followed by a number of oscillations that damp gradually. The whole loop dynamics can be described in terms of exponentially decaying oscillations:

$a(t)=a_{0} \sin (\omega t+\phi) \mathrm{e}^{-t / \tau}$,

where $\tau$ is damping time. The damping may be caused by two mechanisms: energy leakage to surrounding medium (enhanced by curvature and relatively low density contrast of the loop) as well as by the resonant absorption due to the Gaussian profile of the mass density across the loop. However, the resolution of this study is not sufficient to fully consider the effects of resonant absorption. Therefore, energy leakage is left as the main damping mechanism.

Next we perturb AR loops from inside with nearly eigenmode velocity profile. The time signatures are displayed with blue lines in Fig. 4. While in cylindrical loop case we observe 

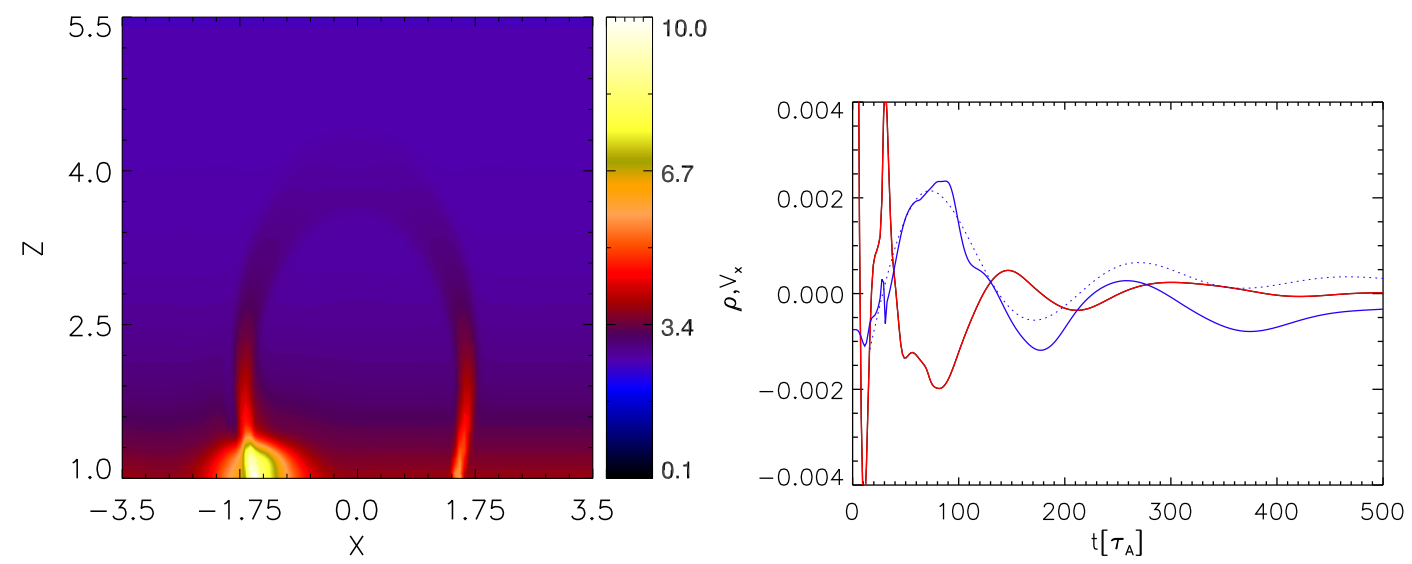

Fig. 5. Left panel: Mass density profiles in $x-z$ plane (for $y=0$ ) at the beginning of simulation, when the pulse appears. Spatial coordinates are measured in units of $L_{S}$. Right panel (from Ofman and Selwa, 2009): Time-signatures of the mass density (red line) and the $x$-component of velocity (blue line) at the loops apex. The dotted line shows the fit used to determine the period and normalized damping time. The density is measured in units of $\varrho_{0}$ and velocity in units of $V_{\mathrm{A} 0}$.

the same scenario as in case of external excitation (exponentially damped harmonic oscillations), the dipole loop shows different dynamics: after the large amplitude initial pulse most of energy leaks to the surrounding plasma causing oscillations of the field which is not uniform. Such a difference in dynamics may result from the fact that collective motion of individual fieldlines that are geometrically identical requires smaller amount of energy to be excited due to the fact that their eigenmode frequencies matches each other. On the other hand, when we excite oscillations in the external field that are not coherent we need more energy at the beginning due to difference in periods and orientation of fieldlines with different eigenmode frequencies (a form of phase mixing). It is noteworthy that during external excitation as the energy trapped inside is not converted to the initial motion of surrounding field, but only serves as a driver of existing oscillation of the field.

Simplified cylindrical models of loops do not consider full variety of AR features, e.g., loops with a range of lengths and orientations and as such can not be used to study dynamics of AR. However, in realistic models of AR loop kink oscillations the excitation should be produced by an external source (e.g. flare).

\subsubsection{Slow standing wave}

Next we add gravity to our model (equations described e.g. in McLaughlin and Ofman, 2008) and slightly change the parameters of the plasma in order to model a hot loop. In order to excite slow standing wave we follow the idea of Selwa et al. (2007b) and launch a pulse in one of the loop's footpoints and in surrounding plasma. The initial stage of the pulse is shown in left panel of Fig. 5. We see clearly that a single footpoint brightening can be observed at the beginning of the event. The scenario corresponds to the observational features reported by Wang et al. (2003b). Both the observational event of 29 September 2000 (10:27 UT) and our numerical simulation start with the strong signal of footpoint brightening.

Following the idea of Selwa et al. (2007b) we launch the pulse in velocity in the way that it covers both the footpoint of the loop and surrounding plasma. After the initial stage the pulse is divided into two components. One component propagates along the loop as a slow pulse until being reflected from the photospheric layer in the opposite footpoint. The other component of the pulse is a fast magnetosonic pulse (which comes from the part of the pulse outside the loop) that propagates across the fieldlines through the ambient corona and reaches the opposite footpoint. As shown by Selwa et al. (2007b) due to the larger fast magnetosonic speed compared to the slow magnetosonic speed, the two parts of the pulse reach both footpoints almost simultaneously (with respect to the time scale of the slow wave period). Such an excitation mechanism of slow standing waves is efficient only for curved loops.

Following Selwa et al. (2007b) we study time signatures of mass density and velocity along the loop at the apex of the loop. Such a position of detection point allows us to measure the amplitudes of fundamental mode and has the advantage that $x$-component of velocity becomes also the longitudinal component at that point. The time signatures are shown in right panel of Fig. 5. We observe the periodic density and velocity fluctuations with the period $P \simeq 160 \tau_{A}$ that after $t \leq 1 P$ reveal quarter wave-period phase-shift indicating slow standing wave. Note, that at the initial stage of the evolution the density and velocity perturbations are in anti-phase which was also observed in coronal loops by Wang et al. (2003a) (right panel of Fig. 5). At the end of simulation the time 
signatures of mass density and velocity are slightly detuned and the period of oscillations starts to vary. This may be the effect of nonlinearity and dissipation. However, as the amplitude of the oscillation drops we are not able to study this effect in detail.

To determine when the standing mode is generated we apply the excitation criterion (Selwa et al., 2005). According to this criterion the standing wave is excited in the system if phase-shift between the mass density and the velocity in the time signatures differs by no more than $20 \%$ from the quarter wave-period. Selwa et al. (2007b) reported the shortest excitation time of the slow standing wave as $1.6 P$ for a $2-$ $\mathrm{D}$ curved loop. The same idea in 3-D geometry leads to the excitation within one wave-period which is fully consistent with observations (Wang et al., 2002, 2003a, b).

From the fit of the damped sine function we find that the damping time is $\tau / P=0.6$. This value is smaller than the observational damping time for loops observed with SUMER (Wang et al., 2002, 2003a, b). Damping in our curved 3-D loop is faster than for the 2-D curved loop $(\tau / P=1.44$, Selwa et al., 2007b). Explanation of this phenomenon in terms of lateral leakage (Selwa et al., 2007b) in all direction is very likely. As plasma $\beta>1$ at the loop's apex, slow waves can easily leave the loop as they as they can propagate obliquely to the field. The 3-D geometry provides additional degrees of freedom for the wave to leak out of the loop (i.e., allowing mode coupling) than 2-D models. Additional amplification of damping comes from the density profile difference (tophat in 2-D study of Selwa et al. (2007b) and Gaussian in 3-D simulation) and lower density ratio of the loop.

A possible extension of the present study could be based on finding more appropriate parameter range for realistic coronal loops, such that the plasma $\beta$ within the loop remains low with height. However, dipole field configuration leads to rapid decrease of magnetic field intensity with height, while in isothermal, gravity-free regime hydrodynamic pressure remains constant, leading to rapid increase of plasma $\beta$ with height. Even when gravity is included, the plasma $\beta$ increases significantly with height in a dipole field configuration (e.g., Ofman and Thompson, 2002). One way to deal with the problem is by choosing very low Euler number (i.e., higher magnetic field at the base of the AR and/or lower plasma temperature). However, in this case slow standing wave excitation time becomes too long in units of the Alfvén time, and difficult to reach in 3-D MHD model with current computational resources. Another possibility is to use a different magnetic field configuration that does not decrease as rapidly with height.

\section{Conclusions}

We model coronal loop oscillations in an active region by means of 3-D MHD simulations. We solve the problem of excitation of vertical and horizontal polarizations of kink loop oscillations. We find that pure vertical mode can be observed if the exciter is launched below the loop. If the pulse acts further away from the loop, both vertical and horizontal components are excited.

We also model two kinds of ARs: curved (more realistic) and straight field with cylindrical loop one. We find that perturbation acting directly on a single loop of AR excites oscillations both in cylindrical and dipole loop. However, the leakage of the wave energy is larger in a curved loop compared to straight loop in the initial phase and remaining amplitude of oscillation is not observed. We find that external excitation of the whole AR is efficient in the excitation of oscillation both in the straight cylindrical AR, and a dipole AR loop and leads to more commonly observed scenario of excitation of AR loop oscillations.

We performed first 3-D MHD simulation of slow standing wave in a curved AR loop. We find that for a curved loop footpoint excitation (with a broader pulse covering not only the footpoint, but also the surrounding plasma) is an efficient mechanism of producing slow standing mode. The main observational features (excitation within one wave-period, initial anti-phase in mass density and velocity signals and initial footpoint brightening) are reproduced by the 3-D MHD model.

Acknowledgements. MS's \& LO's work was financially supported by the NASA SEC Theory program and NASA grants NNG06GI55G, and NNX09AG10G. Computer simulations were performed on Columbia supercomputer at NASA Ames.

Topical Editor R. Forsyth thanks R. Erdelyi and another anonymous referee for their help in evaluating this paper.

\section{References}

Aschwanden, M., Fletcher, L., Schrijver, C., and Alexander, D.: Coronal Loop Oscillations Observed with the Transition Region and Coronal Explorer, The Astrophys. J., 520, 880-894, 1999.

Aschwanden, M. J., De Pontieu, B., Schrijver, C. J., and Title, A. M.: Transverse Oscillations in Coronal Loops Observed with TRACE II. Measurements of Geometric and Physical Parameters, Solar Phys., 206, 99-132, 2002.

Aschwanden, M., Nightingale, R. W., Andries, J., Goossens, M., and Van Doorsselaere, T.: Observational Tests of Damping by Resonant Absorption in Coronal Loop Oscillations, The Astrophys. J., 598, 1375-1386, 2003.

Aschwanden, M., Nakariakov, V., and Melnikov, V.: Magnetohydrodynamic Sausage-Mode Oscillations in Coronal Loops, The Astrophys. J., 600, 458-463, 2004.

Aschwanden, M.: The 3D Geometry, 3D Motion, and Hydrodynamics of Oscillating Coronal Loops, submitted, 2009.

Klimchuk, J. A.: Cross-Sectional Properties of Coronal Loops, Solar Phys., 193, 53-75, 2000.

De Moortel, I., Ireland, J., Walsh, R. W., and Hood, A. W.: Longitudinal intensity oscillations in coronal loops observed with TRACE I. Overview of Measured Parameters, Solar Phys., 209, 61-88, 2002. 
Haynes, M., Arber, T. D., and Verwichte, E.: Coronal loop slow mode oscillations driven by the kink instability, Astron. Astrophys., 479, 235-239, 2008.

López Fuentes, M. C., Klimchuk, J. A., and Démoulin, P.: The Magnetic Structure of Coronal Loops Observed by TRACE, The Astrophys. J., 639, 459-474, 2006.

López Fuentes, M. C., Démoulin, P., and Klimchuk, J. A.: Are Constant Loop Widths an Artifact of the Background and the Spatial Resolution?, The Astrophys. J., 673, 586-597, 2008.

McLaughlin, J. and Ofman, L.: Three-dimensional Magnetohydrodynamic Wave Behavior in Active Regions: Individual Loop Density Structure, The Astrophys. J., 682, 1338-1350, 2008.

Mendoza-Briceño, C. A., Erdélyi, R., and Sigalotti, L. Di G.: The Effects of Stratification on Oscillating Coronal Loops, The Astrophys. J., 605, 493-502, 2004.

Nakariakov, V. M., Ofman, L., Deluca, E. E., Roberts, B., and Davila, J. M.: TRACE observation of damped coronal loop oscillations: Implications for coronal heating, Science, 285(5429), 862-864, 1999.

Nakariakov, V. M., Melnikov, V. F., and Reznikova, V. E.: Global sausage modes of coronal loops, Astron. Astrophys., 412, L7L10, 2003.

Nakariakov, V. M., Tsiklauri, D., Kelly, A., Arber, T. D., and Aschwanden, M. J.: Acoustic oscillations in solar and stellar flaring loops, Astron. Astrophys., 414, L25-L28, 2004.

Ofman, L. and Thompson, B.: Interaction of EIT Waves with Coronal Active Regions, The Astrophys. J., 574, 440-452, 2002.

Ofman, L. and Wang, T. J.: Hot Coronal Loop Oscillations Observed by SUMER: Slow Magnetosonic Wave Damping by Thermal Conduction, The Astrophys. J., 580, L85-L88, 2002.

Ofman, L. and Aschwanden, M. J.: Damping Time Scaling of Coronal Loop Oscillations Deduced from Transition Region and Coronal Explorer Observations, The Astrophys. J., 576, L153L156, 2002.

Ofman, L.: Three dimensional MHD models of active region loops, Adv. Space Res., 36, 1572-1578, 2005.

Ofman, L.: Three-dimensional MHD Model of Wave Activity in a Coronal Active Region, The Astrophys. J., 655, 1134-1141, 2007.

Ofman, L. and Selwa, M.: Three-dimensional MHD modeling of waves in active region loops, Universal Heliophysical Processes, Proceedings of the International Astronomical Union, IAU Symposium, 257, 151-154, 2009.

Ofman, L: Progress, Challenges, and Perspectives of the 3D MHD Numerical Modeling of Oscillations in the Solar Corona, Space Sci. Rev., online, doi:10.1007/s11214-009-9501-1, 2009a.

Ofman, L.: Three-Dimensional Magnetohydrodynamic Models of Twisted Multithreaded Coronal Loop Oscillations, The Astrophys. J., 694, 502-511, 2009 b.

Ogrodowczyk, R. and Murawski, K.: Numerical simulations of slow magnetosonic standing waves in a straight solar coronal slab, Astron. Astrophys., 467, 311-316, 2007.

Patsourakos, S. and Vourlidas, A.: Towards a Better Understanding of CME Onsets with SECCHI on STEREO, AGU Fall Meeting Abstracts, A779, 2007.

Powell, K. G.: An approximate Riemann solver for magnetohydrodynamics (that works in more than one dimension), ICASE Report No. 94-24, Langley, VA, 1994.

Ruderman, M. S. and Roberts, B.: The Damping of Coronal Loop
Oscillations, The Astrophys. J., 577, 475-486, 2002.

Ruderman, M. S. and Erdélyi, R.: Transverse Oscillations of Coronal Loops, Space Sci. Rev., online first, doi:10.1007/s11214-0099535-4, 2009.

Schrijver, C. J., Brown, D. S.: Oscillations in the Magnetic Field of the Solar Corona in Response to Flares near the Photosphere, The Astrophys. J., 537, L69-L72, 2000.

Schrijver, C. J., Aschwanden, M. J., and Title, A. M.: Transverse oscillations in coronal loops observed with TRACE I. An Overview of Events, Movies, and a Discussion of Common Properties and Required Conditions, Solar Phys., 206, 69-98, 2002.

Selwa, M., Murawski, K., and Kowal, G.: Three-dimensional numerical simulations of impulsively generated MHD waves in solar coronal loops, Astron. Astrophys., 422, 1067-1072, 2004.

Selwa, M., Murawski, K., and Solanki, S. K.: Excitation and damping of slow magnetosonic standing waves in a solar coronal loop, Astron. Astrophys., 436, 701-709, 2005a.

Selwa, M., Murawski, K., Solanki, S. K., Wang, T. J., and Tóth, G.: Numerical simulations of vertical oscillations of a solar coronal loop, Astron. Astrophys., 440, 385-390, 2005b.

Selwa, M., Solanki, S. K., Murawski, K., Wang, T. J., and Shumlak, U.: Numerical simulations of impulsively generated vertical oscillations in a solar coronal arcade loop, Astron. Astrophys., 454(2), 653-661, 2006.

Selwa, M., Murawski, K., Solanki, S. K., Wang, T. J.: Energy leakage as an attenuation mechanism for vertical kink oscillations in solar coronal wave guides, Astron. Astrophys., 462, 1127-1135, 2007.

Selwa, M., Ofman, L., and Murawski, K.: Numerical Simulations of Slow Standing Waves in a Curved Solar Coronal Loop, The Astrophys. J., 668, L83-L86, 2007b.

Sigalotti, L. Di G., Mendoza-Briceño, C. A., and Luna-Cardozo, M.: Dissipation of Standing Slow Magnetoacoustic Waves in Hot Coronal Loops, Solar Phys., 246, 187-212, 2007.

Smith, J. M., Roberts, B., and Oliver, R.: Ducted fast waves in coronal loops: curvature effects, Astron. Astrophys., 317, 752$760,1997$.

Taroyan, Y., Erdélyi, R., Doyle, J. G., and Bradshaw, S. J.: Footpoint excitation of standing acoustic waves in coronal loops, Astron. Astrophys., 438, 713-720, 2005.

Terradas, J. and Ofman, L.: Loop Density Enhancement by Nonlinear Magnetohydrodynamic Waves, The Astrophys. J., 610, 523531, 2004.

Tsiklauri, D., Nakariakov, V. M., Arber, T. D., and Aschwanden, M. J.: Flare-generated acoustic oscillations in solar and stellar coronal loops, Astron. Astrophys., 422, 351-355, 2004.

Van Doorsselaere, T., Debosscher, A., Andries, J., and Poedts, S.: The effect of curvature on quasi-modes in coronal loops, Astron. Astrophys., 424, 1065-1074, 2004.

Wang, T. J., Solanki, S. K., Curdt, W., Innes, D. E., and Dammash, I. E.: Doppler Shift Oscillations of Hot Solar Coronal Plasma Seen by SUMER: A Signature of Loop Oscillations?, The Astrophys. J., 574, L101-L104, 2002.

Wang, T. J., Solanki, S. K., Innes, D. E., Curdt, W., and Marsch, E.: Slow-mode standing waves observed by SUMER in hot coronal loops, Astron. Astrophys., 402, L17-L20, 2003a.

Wang, T. J., Solanki, S. K., Curdt, W., Innes, D. E., Dammash, I. E., and Kliem, B.: Hot coronal loop oscillations observed with SUMER: Examples and statistics, Astron. Astrophys., 406, 
1105-1121, 2003b.

Wang, T. J. and Solanki, S. K.: Vertical oscillations of a coronal loop observed by TRACE, Astron. Astrophys., 421, L33-L36, 2004.

Wang, T. J., Solanki, S. K., Innes, D. E., and Curdt, W.: Initiation of hot coronal loop oscillations: Spectral features, Astron. Astrophys., 435, 753-764, 2005.
Wang, T. J., Solanki, S. K., and Selwa, M.: Identification of different types of kink modes in coronal loops: principles and application to TRACE results, Astron. Astrophys., 489, 1307-1317, 2008.

Verwichte, E., Aschwanden, M. J., Van Doorsselaere, T., Foullon, C., and Nakariakov, V. M.: Seismology of a Large Solar Coronal Loop from EUVI/STEREO Observations of its Transverse Oscillation, The Astrophys. J., 698, 397-404, 2009. 